\title{
Postoperative Drains at the Donor Sites of Iliac-Crest Bone Grafts in Patients Who Had a Single Comminuted Long Bone Fracture
}

\author{
Ali Karbalaeikhani ${ }^{1}$, Alireza Saied ${ }^{2}$ \\ ${ }^{1}$ Hand and Plastic Surgery Department, Emam Reza Hospital, \\ AJA University of Medical Sciences, Tehran, Iran \\ ${ }^{2}$ Kerman Neurosceicne Research Center, Kerman University of Medical Sciences, Kerman, Iran \\ E-mail: \{arsaiedmd, aliamousavi\}@yahoo.com \\ Received July 14, 2011; revised October 13, 2011; accepted October 28, 2011
}

\begin{abstract}
In this clinical trial, 90 patients admitted to orthopedics ward, Shahid Mohammadi Hospital, Bandar Abbass with a long bone fracture, comminuted more than 30\%, were randomly divided into two groups. In the first group, after the completion of the operation, a single hemovaccum drain was inserted into the iliac crest wound, the site of cancellous bone removal, whereas the second group didn't receive a drain. The two groups were followed for at least six months and the results were compared with Chi-Square and T-Tests. The two groups, at the end of the follow up period, had no statistically significant difference with regard to pain severity and need for dressing change (in the immediate postoperative period), hematoma formation and infection. So it seems that drain insertion in the wound of patients in whom cancellous bone is removed from the iliac crest, is not necessary.
\end{abstract}

Keywords: Drain, Surgery, Bone Graft, Fracture

\section{Introduction}

Use of devices to evacuate pus and fluids out of the body has been mentioned in Hippocrates records that used a metal tube and wine to evacuate pus from the pleural space of a patient [1]. Drains are used commonly and as a routine procedure after clean surgical operations in hope to prevent hematoma formation and as a result infection occurrence. The rational (but scientifically unproven) reason for this routine has been the hypothesis that a drain would evacuate any oozing blood, serous fluid and forming hematoma from the operation site and so will prevent infection. But this has been a matter of considerable debate: many studies performed on the topic could not prove the ability of drains to prevent hematoma formation [2-4] and aside from this in old [5,6] and new [7] studies drains not only have not prevented infection but have been noticed to be independent risk factors in wound infection after surgery! From a theoretical point of view in addition to prevention of hematoma formation and infection drains would decrease the patients' pain and the need for dressing change in the immediate postoperative days. The aim of this study was to examine the efficacy of drains in the aforementioned subjects (with emphasis on hematoma formation and infection) in one of the common surgical operations in orthopedics, bone graft removal from the iliac crest.

\section{Patients and Methods}

In this clinical trial 102 patients referring to our Hospital emergency room with single comminuted long bone fracture necessitating open reduction and bone graft addition were randomly assigned into Drain (control) and Nondrain (case) groups. The two groups were comparable with regard to age, sex and the broken bone (Table 1).

The patients entering the trial had a single long bone fracture comminuted more than $30 \%$ in whom bone graft was removed from their iliac crest and added to the fracture site during the surgical procedure of open reduction and internal fixation using either plate and screws or intramedullary nails (performed as an open procedure).

Inclusion criteria were as follows: 
Table 1. Fracture distribution of the patients enrolled in the study.

\begin{tabular}{ccccc}
\hline & \multicolumn{2}{c}{ Nondrained } & \multicolumn{2}{c}{ Drained } \\
\cline { 2 - 5 } & female & Male & female & Male \\
\hline Tibia fracture & 6 & 15 & 4 & 18 \\
Femur fracture & 5 & 18 & 2 & 21 \\
humerus fracture & - & - & - & 1 \\
\hline
\end{tabular}

1) Age above 18 years old;

2) Informed consent for entering the trial;

3) Completion of the follow up period of at least 6 months;

4) Absence of systemic disease, pathologic fracture, pregnancy, or chronic medication use;

5) Absence of head, chest or abdominal trauma.

Bone graft was removed from the external iliac crest table in all of the patients and all of the surgical procedures were performed by the senior author. After prep and drape and antibiotic injection (Cephalotine 2 grams intravenous), as the need for bone graft was certain, it was removed first. Using a $10 \mathrm{~cm}$ incision on the iliac crest the muscles were stripped and the cancellous bone was removed by use of osteotome or curette. After the completion of the procedure the wound was irrigated and sutured layer by layer. No attempt was made at hemostasis except for electrocautery. The and in no patient bone wax was used. The surgeon was not aware of the patient's group until this stage when the assistant told him to use or not to use drains before wound closure. In the control group a single hemovacum drain was inserted and sutured to skin. The drain was of the SUPA factory in all of the patients and was removed on the third postoperative day if there was no active drainage. The patients were closely observed for the following during their hospital course and in later follow up in O.P.D. visits:

1) Dressing wetting in the first 24 hours so that the staff had to change the dressing. They were unaware of the study conduction.

2) Pain at the site of bone graft removal so that the patient asked for oral and if nonresponsive for intravenous analgesic. This variable was difficult to measure accurately as the patient may ask for analgesic because of limb pain and this will obscure the pelvic pain.

3) The operation site morbidity (hematoma, infection, serous drainage, infection or any other problem), from the second day after operation up to six months.

The follow up routine in our clinic was as follows: 2 weeks after the operations for wound inspection and suture removal, 4 weeks later for control radiograms and union assessment, 6 weeks later ( 3 months after the operation) for taking control radiograms and probable dis- continuation of limb protection if the union was complete and ultimately 3 months later for assessment of the general condition of the patient and any probable suggestion. In addition the patient was visited out of the program in case of any unexpected complications. In case of infection, oral antibiotics and topical wound care were advised. If there was no or inadequate response, the patient had to be admitted and receive intravenous antibiotics, and if necessary wound irrigation in the operation room under anesthesia would be performed.

The study results were analyzed by computer P4 and SPSS 16 soft ware. The statistical used tests were Chi square and Student's T-Test.

\section{Results}

90 patients entered the trial with the completion of predetermined follow up period, 73 men and 17 women. In total 46 individuals were assigned into Drain and 44 to Nondrain group. The characteristics of both groups are shown in the following tables.

At the end of the follow up period these were the findings:

1) In the Drain group dressing wetting necessitating its change in the first 24 hours didn't occur in any patient, but in 2 of the Nondrain group.

2) In the Drain group 38 needed oral analgesic and 4 needed injection forms, in the Nondrain group 40 needed oral and 2 needed injection analgesic.

3) No patient of either groups developed persistent bleeding or hematoma, although four in the drain group had serosanginous discharge of their wound which had resolved after the first follow up and did not progress to any problem. In the Nondrain group, 2 patients noted severe ecchymosis of their flank up to the mid-thoracic area and their back. Although this caused earlier than scheduled follow up of the patients and their fear, it was apparently harmless and resolved spontaneously without any intervention except reassurance.

4) Three patients developed superficial wound infection, all of the drained group. They noted erythema, pain and warmness in addition to mild discharge of the wound. In all of them the culture was positive for S. aureus and all of them responded to oral antibiotics and local wound care. Deep infection was not observed in any of the patients of the two groups.

Statistical analysis revealed no significant difference between the two groups in any of the aforementioned variables (Table 2).

\section{Discussion}

In this clinical trial we examined the efficacy of drains in 
Table 2. Variables of the study as measured in the two groups.

\begin{tabular}{cccc}
\hline Variable & Nondrained & Drained & p-value \\
\hline Analgesic requirement & 42 & 42 & 0.68 \\
Oral analgesic & 40 & 48 & 0.6 \\
Parenteral analgesic & 2 & 4 & 0.68 \\
Dressing change & 2 & - & 0.24 \\
Hematoma & - & - & - \\
Infection & - & 4 & 0.24 \\
\hline
\end{tabular}

one of the common orthopedic surgical procedures, i.e. bone graft removal from the iliac crest. Although theoretically there is no difference between the wound of iliac crest in patients with different types of surgery on the limbs (for example arthrodesis versus fracture or multiple fractures or nonunion), to lower the chance of bias we determined to limit our study to a very narrow area, namely patients with a single comminuted long bone fracture necessitating bone grafting after open reduction and internal fixation. On the other hand, despite the advances in intramedullary nailing techniques, plating and bone grafting is still mentioned as an acceptable alternative in comminuted long bone fractures and is used in many university hospitals. In addition open intramedullary nailing does not eliminate the need for bone grafts. So we think the study was worthful to be conducted.

From the theoretical point of view a drain is used for the following purposes:

1) Prevention of hematoma as it evacuates any fluid from the wound.

2) Prevention of infection as infection occurs when the hematoma is invaded by bacteria.

3) Decrease in the need for dressing change as the fluid will not "overflow" from the wound.

4) Decrease in the pain that the patient perceives by "decompressing" the wound and lowering its pressure.

Our study could not prove the efficacy of drain in any of the aforementioned.

Surgeons have used drains throughout the history of surgery. No one has any doubt that drain use will be of benefit to the patient if infection is present, but there has been significant controversy about prophylactic use of drains in order to prevent hematoma formation and infection. In fact the advice of pioneers in surgery, about the drain use in clean surgical wounds during the past century has been different from "When in doubt, use drains" [8] to "When in doubt, don't use drains" [1]! Although Hippocrates was probably the first surgeon to use a drain, it was since the era of Andrias Pare and Lister that drains were seriously considered. Pare noticed the adverse effects of the drains and cautioned against overuse of them, but it was until 1880 when a $40 \%$ risk of infection in the drains was discovered. With introduction of modern suction drains several studies on the topic have been undertaken since 1960 [9] and they have resulted in contradictory information. One old study [11] found the risk of infection 3 times less with the use of drains and so anticipated fewer hazards. A study on femur fractures treated by open reduction and internal fixation found the risk of hematoma formation equal in the groups with and without drains, but the risk of infection was obviously greater in the group in which drains were not used [11]. These are probably the only studies that suggest routine drain usage in orthopedic surgery. In fact the largest studies on the topic have been performed on joint arthroplasties and have failed to ascribe any benefit in order to decrease the rate of complications and especially infection and hematoma by drain use in these operations [12-17]. In fact a large metaanalysis revealed increased need for transfusion in patients with drain after arthroplasty [18]!

Few studies have been performed on drain use in operations related to traumatology, but in most of them drain has not proven to be of benefit $[4,19,20]$.

Up to the best of our knowledge only one study has been performed on the topic of drain use in the surgical operation of graft removal from iliac crest [21]. The result was similar to our findings. Drain use did not help in prevention of complications. It is interesting that in neither of these studies even a single case of hematoma was found, as the rate of this complication has been reported to be $3 \%$ [22].

Aside from the unproven benefit, drain use may be associated with complications. In some cases as almost all of us remember, a second operation has been necessary for its removal $[19,22]$.

The serosanginous drainage that was observed after drain removal in some of patients in the present study has been the experience of others too [19] and we have encountered some other cases. Although this was not associated with serious consequences, it was troublesome to the patients.

It must be emphasized that up to the best of our knowledge the latest papers on the topic [23-27]; again did not find drain necessary in orthopedic surgery.

Although based on the present and other studies drain use is unnecessary after surgery, it is difficult to prevent surgeons from a practice that has become a routine and seems rationale: two recent studies [28,29] showed that more than $90 \%$ of surgeons continued to use drains after total knee arthroplasty, despite their awareness of the studies that had not proved the efficacy of drains in these operations. 


\section{References}

[1] M. Levy, "Intraperitoneal Drainage," The American Journal of Surgery, Vol. 147, No. 3, 1984, pp. 309-314. doi:10.1016/0002-9610(84)90156-9

[2] G. W. Varley and S. A. Milner, "Wound Drains in Proximal Femoral Fracture Surgery: A Randomized Prospective Trial of 177 Patients," The Royal College of Surgeons of Edinburgh, Vol. 40, No. 6, 1995, pp. 416-418.

[3] J. Widman, H. Jacobsson, S. A. Larsson and J. Isacson, "No Effect of Drains on the Postoperative Hematoma Volume in Hip Replacement Surgery: A Randomized Study Using Scintigraphy," Acta Orthopaedica Scandinavica, Vol. 73, No. 6, 2002, pp. 625-629. doi:10.1080/000164702321039570

[4] R. M. Tjeenk, M. P. Peeters, E. van den Ende, G. W. Kastelein and P. J. Breslau, "Wound Drainage versus Non-Drainage for Proximal Femoral Fractures. A Prospective Randomised Study,” Injury, Vol. 36, No. 1, 2005, pp. 100-104.

[5] "Factors Influencing the Incidence of Wound Infection," Annals of Surgery, Vol. 160, Supplement 2, 1964, pp. 32-81.

[6] "Report of the Public Health Laboratory Service: Incidence of Surgical Wound Infection in England and Wales,” Lancet, 1960, pp. 659-663.

[7] B. Minnema, M. Vearncombe, A. Augustin, J. Gollish and A. E. Simor, "Risk Factors for Surgical-Site Infection Following Primary Total Knee Arthroplasty,” Infection Control and Hospital Epidemiology, Vol. 25, No. 6, 2004, pp. 477-480. doi:10.1086/502425

[8] M. A. Memon, M. I. Memon and J. H. Donohue, "Abdominal Drains: A Brief Historical Review,” Irish Medical Journal, Vol. 94, No. 6, 2001, pp. 164-166.

[9] J. P. Moss, "Historical and Current Perspectives on Surgical Drainage,” Surgery Gynecology \& Obstetrics, Vol. 152, No. 4, 1981, pp. 517-527.

[10] T. R. Waugh, "Stitch Field FE: Suction Drainage of Orthopedic Wounds," Journal of Bone and Joint Surgery, 1961, Vol. 43A, No. 7, pp. 939-946.

[11] G. W. Varley and S. A. Milner, "Wound Drains in Proximal Femoral Fracture Surgery: A Randomized Prospective Trial of 177 Patients," The Royal College of Surgeons of Edinburgh, Vol. 40, No. 6, 1995, pp. 416-418.

[12] K. J. Beer, A. V. Lombardi Jr., T. H. Mallory and B. K. Vaughn, "The Efficacy of Suction Drains after Routine Total Joint Arthroplasty," The Journal of Bone and Joint Surgery, American Volume, Vol. 73, No. 4, 1991, pp. 584-587.

[13] X. M. Crevoisier, P. Reber and B. Noesberger, "Is Suction Drainage Necessary after Total Joint Arthroplasty? A Prospective Study," Archives of Orthopaedic and Trauma Surgery, Vol. 117, No. 3, 1998, pp. 121-124. doi:10.1007/s004020050210

[14] A. G. D. Valle, G. Slullitel, R. Vestri, F. Comba, M. Buttaro and F. Piccaluga, "No Need for Routine Closed Suction Drainage in Elective Arthroplasty of the Hip: A
Prospective Randomized Trial in 104 Operations,” Acta Orthopaedica Scandinavica, Vol. 75, No. 1, 2004, pp. 30-33. doi:10.1080/00016470410001708050

[15] M. A. Ritter, E. M. Keating and P. M. Faris, "Closed Wound Drainage in Total Hip or Total Knee Replacement. A Prospective, Randomized Study," The Journal of Bone and Joint Surgery, American Volume, Vol. 76, No. 1, 1994, pp. 35-38.

[16] P. J. Walmsley, M. B. Kelly, R. M. Hill and I. Brenkel, "A Prospective, Randomised, Controlled Trial of the Use of Drains in Total Hip Arthroplasty," Journal of Bone and Joint Surgery, British Volume, Vol. 87, No. 10, 2005, pp. 1397-1401. doi:10.1302/0301-620X.87B10.16221

[17] C. Dora, A. von Campe, B. Mengiardi, P. Koch and P. Vienne, "Simplified Wound Care and Earlier Wound Recovery without Closed Suction Drainage in Elective Total Hip Arthroplasty. A Prospective Randomized Trial in 100 Operations," Archives of Orthopaedic and Trauma Surgery, Vol. 127, No. 10, 2007, pp. 919-923. doi:10.1007/s00402-006-0260-0

[18] M. J. Parker, C. P. Roberts and D. Hay, "Closed Suction Drainage for Hip and Knee Arthroplasty. A Meta-Analysis,” The Journal of Bone and Joint Surgery, American Volume, Vol. 86A, No. 6, 2004, pp. 1146-52.

[19] J. P. Cobb, "Why Use Drains?” Journal of Bone and Joint Surgery, British Volume, Vol. 72, No. 6, 1990, pp. 993-995.

[20] G. J. Lang, M. Richardson, M. J. Bosse, K. Greene, R. A. Meyer Jr., S. H. Sims and J. F. Kellam, "Efficacy of Surgical Wound Drainage in Orthopaedic Trauma Patients: A Randomized Prospective Trial,” Journal of Orthopaedic Trauma, Vol. 12, No. 5, 1998, pp. 348-350. doi:10.1097/00005131-199806000-00009

[21] R. C. Sasso, J. I. Williams, N. Dimasi and P. R. Meyer Jr., "Postoperative Drains at the Donor Sites of Iliac-Crest Bone Grafts. A Prospective, Randomized Study of Morbidity at the Donor Site in Patients Who Had a Traumatic Injury of the Spine," The Journal of Bone and Joint Surgery, American Volume, Vol. 80, No. 5, 1998, pp. 631-615.

[22] J. P. Browett, A. N. Gibbs, S. A. Copeland and L. J. Deliss, "The Use of Suction Drainage in the Operation of Meniscectomy,” Journal of Bone and Joint Surgery, British Volume, Vol. 60B, No. 4, 1978, pp. 516-519.

[23] M. J. Parker, V. Livingstone, R. Clifton and A. McKee, "Closed Suction Surgical Wound Drainage after Orthopaedic Surgery," Cochrane Database of Systematic Reviews, 2007, Vol. 18, No. 3, Article ID: CD001825.

[24] L. Cao, N. Ablimit, A. Mamtimin, K. Y. Zhang, G. Q. Li, G. Li and L. B. Peng, "Comparison of No Drain or with a Drain after Unilateral Total Knee Arthroplasty: A Prospective Randomized Controlled Trial,” Chinese Journal of Surgery, Vol. 47, No. 18, 2009, pp. 1390-1393.

[25] R. Clifton, S. Haleem, A. McKee and M. J. Parker, “Closed Suction Surgical Wound Drainage after Anterior Cruciate Ligament Reconstruction: A Systematic Review of Randomised Controlled Trials,” Knee, Vol. 14, No. 5, 2007, pp. 348-351. doi:10.1016/j.knee.2007.07.003 
[26] C. Li, A. Nijat and M. Askar, "No Clear Advantage to Use of Wound Drains after Unilateral Total Knee Arthroplasty: A Prospective Randomized, Controlled Trial,” The Journal of Arthroplasty, Vol. 26, No. 4, 2011, pp. 519-522. doi:10.1016/j.arth.2010.05.031

[27] T. W. Tai, I. M. Jou, C. W. Chang, K. A. Lai, C. J. Lin and C. Y. Yang, "Non-Drainage Is Better than 4-Hour Clamping Drainage in Total Knee Arthroplasty," Orthopedics, Vol. 33, No. 3, 2010, pp. 156-160. doi:10.3928/01477447-20100129-11
[28] A. Chandratreya, K. Giannikas and P. Livesley, “To Drain or Not Drain: Literature versus Practice,” The Royal College of Surgeons of Edinburgh, Vol. 43, No. 6, 1998, pp. 404-406.

[29] S. J. Canty, G. J. Shepard, W. G. Ryan and A. J. Banks, "Do We Practice Evidence Based Medicine with Regard to Drain Usage in Knee Arthroplasty? Results of a Questionnaire of BASK Members,” Knee, Vol. 10, No. 4, 2003, pp. 385-387. doi:10.1016/S0968-0160(03)00037-1 